\title{
BMJ Open Comparison of high-flow nasal oxygen therapy and non-invasive ventilation in ICU patients with acute respiratory failure and a do-not-intubate orders: a multicentre prospective study OXYPAL
}

René Robert (10 , ${ }^{1,2}$ Denis Frasca, ${ }^{3}$ Julie Badin, ${ }^{4}$ C Girault, ${ }^{5}$ Christophe Guitton, ${ }^{6}$ Michel Djibre, ${ }^{7}$ Pascal Beuret, ${ }^{8}$ Jean Reignier, ${ }^{9}$ Dalila Benzekri-Llefevre, ${ }^{10}$ Suela Demiri, ${ }^{11,12}$ Hassène Rahmani, ${ }^{13}$ Laurent Argaud Argaud, ${ }^{14}$ Erwan I'her (10 , ${ }^{15,16}$ Stephan Ehrmann, ${ }^{17}$ Olivier Lesieur, ${ }^{18}$ Khaldoune Kuteifan, ${ }^{19}$ Francois Thouy, ${ }^{20}$ Laura Federici, ${ }^{21}$ Didier Thevenin, ${ }^{22}$ Damien Contou, ${ }^{23}$ Nicolas Terzi, ${ }^{24}$ Saad Nseir, ${ }^{25}$ Martial Thyrault, ${ }^{26}$ Christophe Vinsonneau, ${ }^{27}$ Juliette Audibert, ${ }^{28}$ Juliette Masse, ${ }^{29}$ Alexandre Boyer, ${ }^{30}$ Bertrand Guidet, ${ }^{31}$ Riad Chelha, ${ }^{32}$ Jean-Pierre Quenot, ${ }^{33}$ G Piton, ${ }^{34}$ Nadia Aissaoui, ${ }^{35}$ Arnaud W Thille, ${ }^{1,2}$ Jean-Pierre Frat ${ }^{1,2}$

To cite: Robert R, Frasca D, Badin J, et al. Comparison of high-flow nasal oxygen therapy and non-invasive ventilation in ICU patients with acute respiratory failure and a do-not-intubate orders: a multicentre prospective study OXYPAL. BMJ Open 2021;11:e045659. doi:10.1136/ bmjopen-2020-045659

- Prepublication history for this paper is available online. To view these files, please visit the journal online (http://dx.doi. org/10.1136/bmjopen-2020045659).

Received 08 0ctober 2020 Revised 18 November 2020 Accepted 19 November 2020

Check for updates

(c) Author(s) (or their employer(s)) 2021. Re-use permitted under CC BY-NC. No commercial re-use. See rights and permissions. Published by BMJ.

For numbered affiliations see end of article.

Correspondence to Professor René Robert; rene.robert@chu-poitiers.fr

\section{ABSTRACT}

Introduction A palliative approach to intensive care unit (ICU) patients with acute respiratory failure and a do-notintubate order corresponds to a poorly evaluated target for non-invasive oxygenation treatments. Survival alone should not be the only target; it also matters to avoid discomfort and to restore the patient's quality of life. We aim to conduct a prospective multicentre observational study to analyse clinical practices and their impact on outcomes of palliative high-flow nasal oxygen therapy (HFOT) and non-invasive ventilation (NIV) in ICU patients with do-not-intubate orders.

Methods and analysis This is an investigator-initiated, multicentre prospective observational cohort study comparing the three following strategies of oxygenation: HFOT alone, NIV alternating with HFOT and NIV alternating with standard oxygen in patients admitted in the ICU for acute respiratory failure with a do-not-intubate order. The primary outcome is the hospital survival within 14 days after ICU admission in patients weaned from NIV and HFOT. The sample size was estimated at a minimum of 330 patients divided into three groups according to the oxygenation strategy applied. The analysis takes into account confounding factors by modelling a propensity score. Ethics and dissemination The study has been approved by the ethics committee and patients will be included after informed consent. The results will be submitted for publication in peer-reviewed journals.

Trial registration number NCT03673631

\section{INTRODUCTION}

Background and rationale

In intensive care unit (ICU) patients with acute respiratory failure, non-invasive strategies of oxygenation are mainly used to avoid

\section{Strengths and limitation of this study}

- This trial will be the largest prospective study specifically targeted on palliative use of non-invasive oxygenation strategies in patients admitted to intensive care unit for acute respiratory failure with do-notintubate order.

- The study will also include patients who have been intubated and then developed post-extubation respiratory failure with a do-not-reintubate order. There are currently very limited data concerning this patient subgroup.

- To analyse the benefit of such therapies in a palliative context, specific attention will be paid to patientcentred outcomes: tolerance, comfort, intermediate and long-term evolution of quality of life

- Given the heterogeneity of clinical situations, and more specifically the presence or absence of hypercapnia, the study has not been randomised. The study aims to evaluate bedside practice and to take into account the effect of confounding factors, and a propensity will be calculated individually for each treatment.

invasive mechanical ventilation. ${ }^{1}$ A palliative approach to some ICU patients with acute respiratory failure corresponds to a specific target for non-invasive oxygenation treatments. ${ }^{2-4}$ In such situations, the goal of treatment should not only be survival, but also improved respiratory condition and avoidance of inappropriate discomforts. Medium-term or long-term benefits should be evaluated in 
view of assessing the actual benefit of these techniques under the above-mentioned conditions. ${ }^{56}$ Only rarely in ICUs, do non-invasive palliative oxygenation strategies correspond to compassionate treatment, of which the only goal should be to reduce discomfort related to dyspnoea. ${ }^{4}$ In most cases, non-invasive palliative oxygenation strategies are applied in selected patients with the hope of clinical recovery for whom endotracheal ventilation is not an option, but with the hope of clinical recovery. Similarly, in some patients who have been initially intubated and mechanically ventilated, the do-not-reintubate question may arise. Schematically, two types of patients may benefit from these non-invasive approaches: patients with severe chronic obstructive pulmonary disease (COPD) or chronic heart failure and patients with cancer or haematologic malignancy. While previous studies in the first group have reported that nearly half survive and can return home, ${ }^{4}$ for patients with malignancies, the prognosis is more severe. ${ }^{47}$

There has been rapidly increasing interest in high-flow nasal oxygen therapy (HFOT), which has been reported in different clinical situations to be more effective and better tolerated than non-invasive ventilation (NIV) ${ }^{89}$ However, results are conflicting and controversies about its possible superiority have remained persistent. ${ }^{10-13}$ Frequency of use of non-invasive oxygenation strategies in ICU patients with do-not-intubate order is not precisely known. In these palliative ICU patients, the balance between benefits and harms of the non-invasive oxygenation supports has been inadequately investigated especially with regard to HFOT, and the impact on medium-term or long-term quality of life has yet to be studied. ${ }^{1}$

\section{Objectives}

We aim to conduct a prospective multicentre observational study comparing the practices and impact of palliative non-invasive oxygenation strategies, including HFOT and NIV, in patients admitted to an ICU for acute respiratory failure with a do-no-intubate order. We hypothesised that a strategy involving HFOT alone is more beneficial in terms of survival and comfort compared with strategies of NIV alternating with standard oxygen or HFOT.

\section{Primary objective}

To compare the survival within 14 days of ICU admission among patients with acute respiratory failure and a do-nointubate order alive and weaned from an oxygenation strategy using high-flow nasal oxygen alone versus NIV alternating with standard oxygen and NIV with high-flow nasal oxygen.

\section{Secondary objectives}

To compare the three strategies of oxygenation as regards:

- Short-term efficacy of the non-invasive oxygenation strategies.

- Tolerance, acceptance comfort and sleep quality under the non-invasive strategies of oxygenation during ICU stay.
- Length of stay in ICU and hospital, mortality rates in ICU, in hospital, at day 28 and up to day 90 , and the number of decision to withdraw life-support decision.

- Quality of life in survivors at 3 and 6 months after ICU discharge.

\section{Trial design}

The OXYPAL study is an investigator initiated, prospective multicentre, observational study comparing non-invasive strategies of oxygenation using HFOT alone versus NIV alternating with standard oxygen and with HFOT in ICU patients with severe acute respiratory failure and do-notintubate orders.

\section{METHODS \\ Participants, interventions and outcome \\ Study setting}

The OXYPAL study is taking place in 34 mixed medical and surgical ICUs in France.

\section{Eligibility criteria}

\section{Inclusion criteria}

Adult patients admitted to ICU with acute respiratory failure and a do-not-intubate order will be eligible. Acute respiratory failure is defined as a respiratory rate above 25 breaths/minute or signs of respiratory distress, and hypoxemia requiring more than $3 \mathrm{~L} /$ min oxygen flow to obtain pulse oximetry above $92 \%$.

Two types of patients will be considered at inclusion according to the following criteria: patients with acute respiratory failure requiring non-invasive strategies of oxygenation within the 24 hours of admission and patients initially mechanically ventilated with postextubation respiratory failure (figure 1 ).

\section{Exclusion criteria}

Refusal to participate, patients with home care treatment by NIV, patients treated with prophylactic HFOT or NIV after extubation.

\section{Intervention}

Patients will be included after obtaining their informed consent or from their next of kin, and non-invasive strategies of oxygenation will then be carried out within the 24 hours after they meet inclusion criteria. The purpose of the 24-hour time frame is to allow confirmation of the do-not-intubate orders, including collegiality of the decision according to French law on withholding or withdrawing therapies.

The non-invasive oxygenation strategy to be applied will be left at the appreciation of the physician in charge of the patient (HFOT alone or NIV alternating with HFOT or NIV alternating with standard oxygen). In line with the recent international guidelines, ${ }^{1}$ NIV will be privileged in hypercapnic patients with underlying chronic lung disease.

Three groups of patients will be determined according to the non-invasive strategy of oxygenation carried out: 


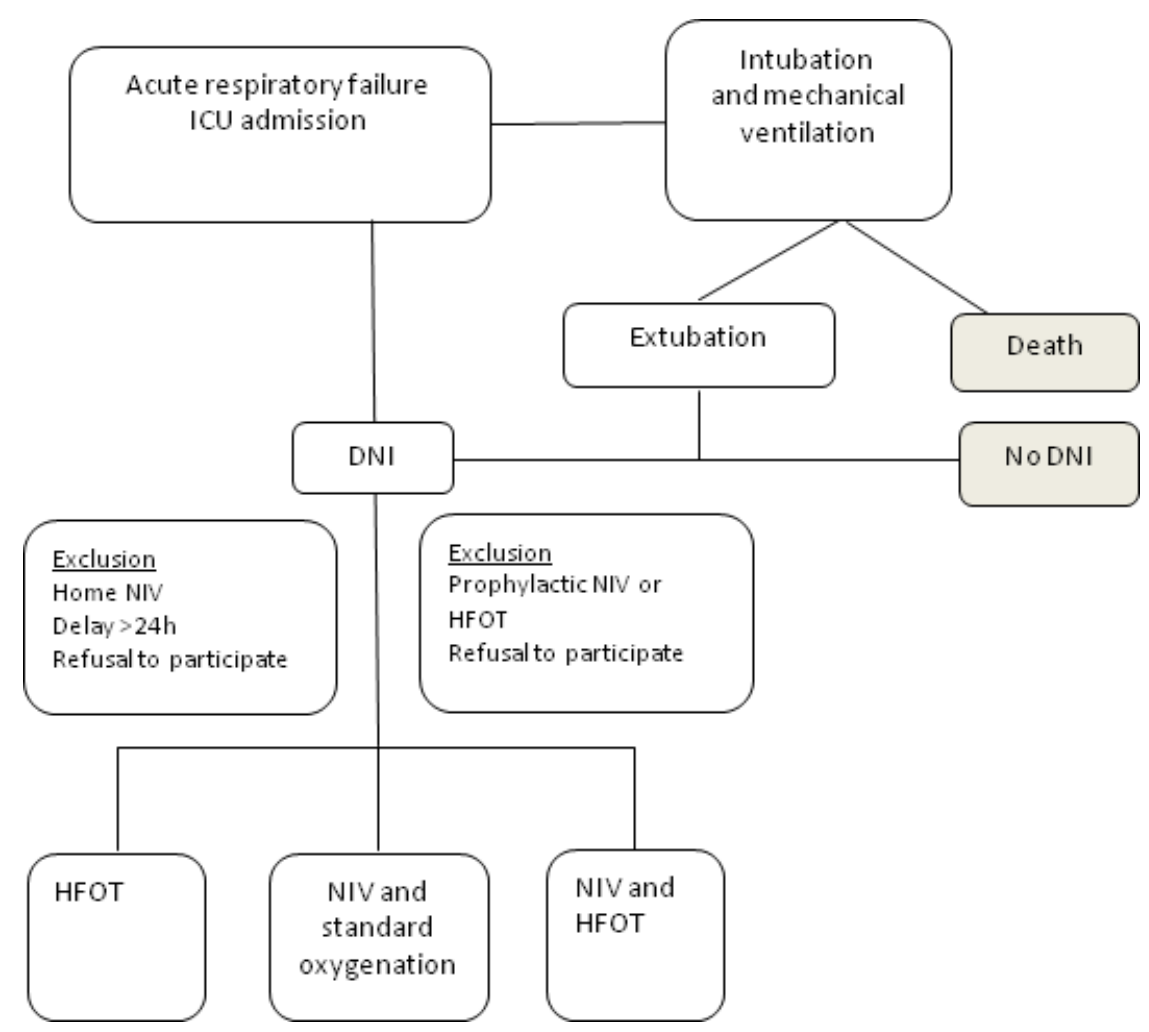

Figure 1 Flowchart of the patients and study design. DNI, do-not-intubate; HFOT, high-flow oxygen therapy; ICU, intensive care unit; NIV, non-invasive ventilation.

continuous HFOT, sessions of NIV interspaced with standard oxygen therapy and sessions of NIV interspaced with HFOT.

To standardise homogenate practices, basic recommendations in accordance with guidelines are specified in the study protocol

\section{HFOT}

HFOT (Optiflow or AIRVO2, Fisher \& Paykel, Auckland, New Zealand) is continuously administered with a gas flow of at least $40 \mathrm{~L} / \mathrm{min}$ and $\mathrm{FiO}_{2}$ adjusted to obtain adequate oxygenation (pulse oximetry of at least 92\%) through a heated humidifier (MR 850, Fisher \& Paykel, Auckland, New Zealand) set to the 'intubation' position.

\section{NIV}

NIV is carried out with a ventilator dedicated for NIV (ICU ventilator after activation of NIV mode or NIV bi-level ventilator ${ }^{10}$ in pressure support (PS) ventilatory mode with a minimal PS level of $5 \mathrm{~cm} \mathrm{H}_{2} \mathrm{O}$ targeting a tidal volume of $6 \mathrm{~mL} / \mathrm{kg}$ of predicted body weight and avoiding tidal volumes exceeding $8 \mathrm{~mL} / \mathrm{kg}$, a positive end-expiratory pressure (PEEP) level of at least $8 \mathrm{~cm}$ $\mathrm{H}_{2} \mathrm{O}$, and $\mathrm{FiO}_{2}$ adjusted to obtain adequate oxygenation (pulse oximetry of at least 92\%). For patients experiencing NIV intolerance despite reassurance, physicians will be encouraged to modify NIV settings (level of PS and PEEP, minimisation of leaks, adjustment of inspiratory trigger and cycling, and interface switch) to improve NIV tolerance.
As this observational study will correspond to bedside practice, while no minimal duration/day will be imposed by the protocol, a first session of at least 2 hours and further sessions lasting at least 1 hour are to be encouraged.

Standard oxygen

In the group with NIV sessions interspaced with standard oxygen therapy, oxygen therapy is applied continuously through a non-rebreather face mask at a flow rate adjusted to maintain an oxygen saturation level of $92 \%$ or more, as measured by means of pulse oximetry $\left(\mathrm{SpO}_{2}\right)$.

In the three groups, continuation or discontinuation of the treatment will be decided according to patient's respiratory status. Reasons for discontinuation, that is, improvement, intolerance or refusal of the oxygenation strategy, will be recorded on the case report form.

\section{Definitions}

Active palliative oxygenation support is given to patients provided that the cause of acute respiratory failure is reversible and oxygenation support improves patient comfort. ${ }^{14}$ Compassionate oxygenation support is given to patients receiving only comfort care alone to alleviate their dyspnoea.

\section{Outcomes}

Primary outcome

The primary outcome is survival in patients weaned from HFOT and NIV within day 14 after ICU admission. 


\section{Secondary outcomes}

Secondary outcome variables include the following:

- Short-term efficacy will be evaluated by a decrease in respiratory rate below 20 breaths/min, pulse oximetry above $92 \%$ and Borg scale scores.

- Tolerance will be evaluated by $100-\mathrm{mm}$ visual analogue scale from 'no discomfort' to 'maximal imaginable discomfort' and by identification of the reason of eventual discontinuation of the noninvasive oxygenation strategy; quality of sleep will be evaluated semi-quantitatively by the patient himself and by the nurse in charge of the patient during his or her nightshift, with a Likert scale: (good, somewhat good slightly good and not good). Overall satisfaction at ICU discharge and global acceptance of the oxygenation method applied will be expressed at the end of the ICU stay by the patient, a patient's relative and the nurse in charge of the patient by two distinct Likert scales: very satisfied, satisfied, somewhat satisfied, and unhappy and extremely unhappy; and useful and easy to accept, useful but hard to accept, useless but easy to accept, and useless and hard to accept.

- Length of stay in ICU and in hospital, survival rates in ICU and in hospital, at day 28 and up to day 90 , and the number of decisions to withdraw life support decision.

- Quality of life will be assessed by the Euro Quality of life 5 dimensions (EQ5D) score recorded by phone call at 3 and 6 months.

\section{Participant timeline}

Table 1 shows assessment and visits for participants.

\section{Sample size}

This observational study will be constituted by three groups: HFOT alone, NIV with standard oxygen, and NIV with HFOT, according to the strategy initially chosen by the clinicians. We assume proportions of surviving patients weaned from non-invasive strategies of oxygenation within day 14 of $50 \%$ in the NIV group and $65 \%$ in the HFOT group. There do not exist enough data to speculate on survival in the NIV associated with the HFOT group. Considering the proportions of patients receiving each strategy of oxygenation, $30 \%$ for HFOT, $60 \%$ for NIV with standard oxygen and $20 \%$ for NIV with HFOT (the total exceeds $100 \%$ to take account of the variability of the proportions), for a power of $80 \%$ and a type I error of $5 \%$, for the relative hazard of 0.65 , the number of patients required is at least 70 in the HFOT alone group, 130 in the NIV with standard oxygen group and 40 in the NIV with HFOT group, that is, a total of 240 patients to estimate mortality hazard with precision of $1 \%$. The control of confounding factors will be carried out by the inverse weighting method of propensity score. Adjusting the sample size for the propensity score and possible non-exploitable records, the number of patients required is 300 (80 patients in HFOT alone group, 170 in NIV with standard oxygen group and 50 in NIV with HFOT group), that is, recruitment of 8-15 patients/year for a target of 34 participating centres. Sample size has been increased to 330 to account for the inclusion of patients on home NIV (addition of the noninclusion criterion after the first inclusions).

\section{Blinding}

As allocation of the non-invasive oxygenation strategy is based on a not masked practitioner's approach, the statistician and the co-ordinating centre investigators will

\begin{tabular}{|c|c|c|c|c|c|c|c|c|c|}
\hline Actions & Baseline & D1 & D2 & D3 & D14 & ICU discharge & Hospital discharge & M3 & M6 \\
\hline Inclusion/exclusion criteria & $x$ & & & & & & & & \\
\hline Informed consent & $\mathrm{X}$ & & & & & & & & \\
\hline Group attribution & $x$ & & & & & & & & \\
\hline \multicolumn{10}{|l|}{ Analysis } \\
\hline Dyspnoea score & $x$ & & & & & & & & \\
\hline Respiratory rate & $\mathrm{x}$ & & & & & & & & \\
\hline Comfort scale with oxygenation method & & $\mathrm{X}$ & $\mathrm{x}$ & $x$ & & & & & \\
\hline \multicolumn{10}{|l|}{ Quality of sleep (patient) } \\
\hline \multicolumn{10}{|l|}{ Quality of sleep (nurse) } \\
\hline Sedation $(y / n)$ & & $\mathrm{x}$ & $\mathrm{x}$ & $\mathrm{x}$ & $x$ & & & & \\
\hline NIV duration & & $\mathrm{x}$ & $\mathrm{x}$ & $x$ & $x$ & & & & \\
\hline HFOT & & $\mathrm{x}$ & $\mathrm{x}$ & $\mathrm{x}$ & & & & & \\
\hline Outcome (death/alive) & & & & & $x$ & $x$ & $x$ & $x$ & $x$ \\
\hline ED5Q & $X^{*}$ & & & & & & & $x$ & $x$ \\
\hline
\end{tabular}

${ }^{*} \mathrm{ED} 5 \mathrm{Q}$ at baseline corresponds to quality of life before the current admission.

HFOT, high-flow nasal oxygen therapy; ICU, intensive care unit; NIV, non-invasive ventilation. 
remain unaware of the study group outcomes until the data are locked.

\section{Recruitment}

Current status: the first patient was included on 15 October 2018.

Due to inclusion curve, and study recruitment discontinuation during the COVID-19 period (March to May 2020), amendment has been provided for a 6-month prolongation of inclusion period. The expected date of complete data collection has been set: mid-April 2021

(6-month follow-up of the last patient included).

1. Expected date of the end of monitoring of participating centres: June 2021.

2. Expected starting date of data analysis: last trimester 2021.

\section{Assignment of intervention, data collection, management and analysis \\ Assignment of intervention}

Allocation of the non-invasive strategy of oxygenation is based on the practitioner's approach in accordance with the recent guidelines ${ }^{1}$ leading to three groups of treatment: HFOT alone, NIV with standard oxygen and NIV with HFOT.

\section{Data collection and management}

Data will be collected on an electronic case report form by a trained investigator or research assistant at each centre. At time of inclusion, the following data will be collected:

- Demographic characteristics : age, sex, simplified acute physiology score II, body mass index, cause of acute respiratory distress, comorbidities: diabetes, cirrhosis, stroke with motor sequelae, cognitive impairment (dementia), chronic psychiatric illness, depressive syndrome, coronary artery disease, New York Heart Association (NYHA) IV heart failure, stage IV COPD, pulmonary fibrosis, progressive cancer and progressive haematological malignancy.

- Reasons for the decision not to intubate: patient refusal, cancer, malnutrition, heart failure NYHA IV and severe chronic respiratory failure.

- Variables related to clinical efficacy on the first day: respiratory rate on admission and 1 hour after the first technique used, $\mathrm{SpO} 2$ before and 1 hour after the first technique used, blood gas and chest X-ray before treatment (if performed). On the following days: respiratory rate and SpO2. Processing monitoring variables (daily): duration of NIV per day, duration of HFOT per day, and in the event of a change in the initially planned strategy, reason for the change.

- Sleep quality during the first night (patient's estimation and perception of sleep quality by patient's nurse). On the following days: sleep quality on second and third night, perception of sleep quality, possible introduction of sedation (and nature of sedation) and treatment received by the patient (hypnotics, anxiolytics, morphine and neuroleptics), overall satisfaction at ICU discharge and global acceptance of the used oxygenation method scales used.

- Quality-of-life variables: EQ5D score prior to ICU hospitalisation and at 3 and 6 months.

\section{Statistical methods}

Descriptive analysis of patients groups at baseline

Categorical variables will be presented as frequencies and percentages, and continuous variables as means with SD or medians with IQR (25-75 percentile). Comparison of continuous data between groups will be carried out using the unpaired t-test and categorical data will be compared using the $\chi^{2}$ test.

\section{Analysis pertaining to the main criteria of evaluation}

For the primary endpoint, analysis of survival in patients weaned from oxygenation support within day 14 will be compared by Kaplan-Meyer estimator and log-rank test. To adjust in-hospital survival for confounding factors, including demographic variables, comorbidities, disease severity and other medications used, a Cox model proportional hazards regression will be performed. From the model, HRs with 95\% CIs will be estimated for included variables to determine their effect on the risk of mortality (primary endpoint).

Proportionality between the predictors and the HR will be validated through evaluation of Schoenfeld residuals with a $\mathrm{p}>0 \cdot 05$, confirming proportionality.

To minimise the effect of confounding factors, a propensity score matching analysis by the inverse weighting method will be carried out individually for each of the HFOT alone and NIV with HFOT groups compared with NIV with standard oxygen group. In the event of a change in treatment strategy, the patient will be analysed in the originally initiated treatment group.

All the analyses will be performed by the study statistician according to a predefined statistical analysis plan and using statistical software (SAS V.9.4 or R software). For statistical tests, a two-tailed $\mathrm{p}$ value of $<0.05$ will be considered as indicating statistical significance.

\section{Analysis pertaining to the main criteria of evaluation}

Secondary endpoints will be compared between the three treatment groups by means of analysis of variance (or non-parametric method, if appropriate) for quantitative variables and by means of the $\chi^{2}$ test (or Fisher's exact test) for qualitative variables.

\section{Predetermined subgroup analysis}

Subgroup analysis will be conducted to analyse outcomes in patients with and without hypercapnia and in patients with and without malignancy.

To take into account patients' tolerance of oxygenation support systems, subgroup of patients who could complete the prescribed treatment will be compared with patients who refused NIV or HFNT whatever the time elapsed between initiation and refusal. 


\section{ETHICS AND DISSEMINATION}

\section{Consent or assent}

The study was approved by an independent ethics committee (Comité de protection des personnes Ile de France V for number ID-RCB-2017-A03034-49). The University Hospital of Poitiers is the sponsor of the trial. Patients will be included after verification of the eligibility criteria and after having addressed an informed consent form to the investigator according to the decision of the central ethics committee. For patients not able to provide informed consent, their next-of-kin will be contacted according to the same procedure. Patients will be informed as soon as possible by the investigator of their participation in the study and their consent to continue to participate in the study will be retrieved. Patients will be informed that they will be called 3 and 6 months after ICU discharge for evaluation of their quality of life using the EQ5D score.

\section{Confidentiality}

Data will be handled according to French law. All original records will be archived at trial sites for 25 years. The clean database file will be anonymised and kept for 15 years.

\section{Declaration of interest}

The OXYPAL study is an investigator-initiated trial. The European research network REVA has endorsed the study project. The study is promoted by the University Hospital of Poitiers. The study promoter has received grants from 'Fondation de France', 'AADAIRC' and 'Association Sports et Collection'. Grant numbers are not applicable.

\section{Access to data}

All investigators will have access to the final data set. Participant-level data sets will be made accessible on a controlled access basis.

\section{Dissemination policy}

Findings will be published in peer-reviewed journals and presented at local, national and international meetings, and conferences to publicise and explain the research to clinicians, commissioners and service users.

\section{DISCUSSION}

There is little knowledge about the proportion of patients admitted at an ICU with acute respiratory failure and do-not-intubate orders and even less information about not to be re-intubate orders. ${ }^{15}$ Thirteen studies have reported data on patients receiving $\mathrm{NIV}^{5-7}{ }^{16-25}$ three receiving HFOT and two comparing NIV and HFOT. ${ }^{26-30}$ The main findings of these studies are the following: (1) two main types of patients are considered, patients with active cancer or haematologic malignancies and those patients with exacerbation of chronic respiratory disease or acute on chronic heart failure; ${ }^{61}$ (2) the hospital mortality rate of the second category is around $50 \%$, and it is higher in patients with cancer; ${ }^{6713}$ and (3) non-invasive strategies of oxygenation enable respiratory improvement in a large proportion of patients. ${ }^{6} 7212627$ When survival is identified as a primary objective in ICU patients, day 28 is almost always the gold standard choice. Considering the high mortality rate and the relatively short length of stay of the patients having declined tracheal intubation, ${ }^{6}$ as regards the primary objective, we focused on in the first 14 days of hospitalisation. Additionally, numerous points warrant specific investigations. Importantly, in these palliative patients, the expected benefit of non-invasive techniques of oxygenation should not be reduced to immediate efficacy and mortality rates, but should also include patient-centred outcomes such as comfort, acceptance and tolerance of these techniques and surviving patients' evaluation of their quality of life. ${ }^{32}$

NIV is assumed to be generally well-tolerated by patients with do-not-intubate orders in only one study. ${ }^{31}$ In largescale studies, NIV has been discontinued in 20\%-25\% of patients due to poor tolerance. ${ }^{83}$ In patients with comfort measurements, only $10 \%$ stopped NIV treatment because of intolerance or adverse effects. ${ }^{6} 7$ It should also be pointed out that $34 \%$ of patients reported regrets about having received NIV in a full code strategy context. ${ }^{34}$ HFOT is generally well-tolerated. ${ }^{8}$ Quality of sleep is a major challenge when assessing in ICU patients' comfort and it has been shown that sleep deprivation may be associated with altered respiratory performance. ${ }^{35}$ Only one study has reported poor sleep during NIV in $76 \%$ of the studied patients. ${ }^{36}$ In the OXYPAL study, particular attention will be paid to quality of sleep in all the included patients.

The quality of life of survivors with do-not-intubate orders was measured in two studies only reporting similar Short Form Health Survey and Hospital Anxiety and Depression Scale (HADS) scores compared with patients receiving NIV in full-code strategy ${ }^{6} 36$

The OXYPAL study will be the first large prospective study focused on bedside practice of non-invasive oxygenation in patients admitted to ICU with DNI orders, including patients with do-not reintubate orders. This study will analyse not only outcome, but also the tolerance and the quality of life at short and medium time. A limitation will be due to the observational study design, and we cannot exclude the possibility of unmeasured confounding factors, although the propensity scorematched analyses will ensure comparability on measured confounding factors.

Although it is not a randomised study, this study will give some indication with the respective potential benefit of the different used strategies and we should be able to suggest the preferred method in different clinical circumstances.

\section{Author affiliations}

${ }^{1}$ Médecine Intensive Réanimation, Centre Hospitalier Universitaire de Poitiers, Poitiers, France

${ }^{2}$ ALIVE Research Group, CIC 1402 INSERM, University of Poitiers, Poitiers, France ${ }^{3}$ Methods in Patient-Centered Outcomes and Health Research, INSERM UMR1246, Poitiers, France 
${ }^{4}$ Service de Réanimation Médico-Chirurgicale, Blois, France, Centre Hospitalier de Blois, Blois, France

${ }^{5}$ Université de Rouen,CHU de Rouen,Service de Réanimation Médicale, Rouen

University Hospital, Rouen, France

${ }^{6}$ Service de Réanimation Médico-Chirurgicale et Unité de Surveillance Continue, Centre Hospitalier Le Mans, Le Mans, France

${ }^{7}$ Service de Médecine Intensive Réanimation, Hôpital Tenon, APHP, Sorbonne Université, Paris, France

${ }^{8}$ Service de Réanimation et Soins Continus, Centre Hospitalier de Roanne, Roanne, France

${ }^{9}$ Medecine Intensive Réanimation, Université de Nantes, CHU de Nantes, Nantes,

Pays de la Loire, France

${ }^{10}$ Service de Réanimation Polyvalente, Centre Hospitalier Régional, Hopital de la Source, Orleans, France

${ }^{11}$ Service de Pneumologie, Médecine intensive - Réanimation (Département "R3S"), AP-HP. Sorbonne Université, Hôpital Pitié-Salpêtrière, Paris, France

${ }^{12}$ INSERM, UMRS1158 Neurophysiologie Respiratoire Expérimentale et Clinique,

Sorbonne Université, Paris, France

${ }^{13}$ Service de Réanimation Médicale, Université de Strasbourg, CHU de StrasbourgHopital Civil, Strasbourg, France

${ }^{14}$ Service de Médecine Intensive Réanimation, Université de Lyon, Lyon, France

${ }^{15}$ Médecine Intensive et Réanimation, CHRU de Brest, Brest, France

${ }^{16}$ LATIM INSERM UMR 1101, Université de Bretagne Occidentale, Brest, France

${ }^{17}$ Médecin Intensive Réanimation, CIC 1415, CRICS-TriggerSEP, Centre d'Étude des

Pathologies Respiratoires, INSERM U1100, Université de Tours, CHU de Tours, Tours, France

${ }^{18}$ Service de Réanimation Polyvalente, Centre Hospitalier Saint Louis, La Rochelle, France

${ }^{19}$ Service de Réanimation Médicale, Centre Hospitalier Mulhouse, Hopital Emile Muller, Mulhouse, France

${ }^{20}$ Service de Réanimation Médicale, Université de Clermont-Ferrand,CHU Gabriel

Montpied, Clermont-Ferrand, France

${ }^{21}$ Service de Réanimation Médico-Chirurgicale, Centre Hospitalier Louis Mourrier, Colombe, France

${ }^{22}$ Service de Réanimation Polyvalente, Centre Hospitalier de Lens, Lens, France

${ }^{23}$ Service de Réanimation Polyvalente, Centre Hospitalier Victor Dupouy, Argenteuil,

France

${ }^{24}$ Service de Réanimation Médicale, Université de Grenoble, CHU Grenoble, Grenoble, France

${ }^{25}$ Crit Care, University Hospital of Lille, Lille, France

${ }^{26}$ Service de Réanimation Polyvalente, Groupement Hospitalier Nord Essonne, Longjumeau, France

${ }^{27}$ Service de Réanimation Polyvalente et USC, Centre Hospitalier Bethune Beuvry, Bethune, France

${ }^{28}$ Service de Réanimation Polyvalente et USC, Hopital Louis Pasteur, Chartres, France

${ }^{29}$ Service de Médecine Intensive Réanimation, Université Catholique de Lille, Lille,

France

${ }^{30}$ Service de Réanimation Médicale, Université de Bordeaux, CHU de Bordeaux Groupe Hospitalier Pellegrin, Bordeaux, France

${ }^{31}$ Service de Médecine Intensive Réanimation, CHU Saint-Antoine, Paris, France

${ }^{32}$ Service de Réanimation Médicale, Hopital Privé Claude Galien, Quincy, France

${ }^{33}$ Réanimation Médicale, CHU Dijon, Dijon, France

${ }^{34}$ Service de Medecine Intensive Réanimation, Université Bourgogne-FrancheComté; CHU Besançon - Hopital Jean Minjoz, Besançon, France

${ }^{35}$ Service de Médecine Intensive Réanimation, Hopital Europeen Georges Pompidou, Paris, France

\section{Twitter Laura Federici @laurafederici and Jean-Pierre Quenot @Quenot}

Acknowledgements The authors wish to thank Jeffrey Arsham for reviewing and editing the original English-language manuscript.

Contributors RR, JP-F and AWT in collaboration with all authors designed the study and wrote the manuscript together. DF provided substantial contributions to the conception and design of the study, and wrote the statistical analysis plan and estimated the sample size. RR, DF, JB, CG, CG, MD, PB, JR, DB-L, SD, HE, LAA, El, SE, OL, KK, FT, LF, DT, DC, NT, SN, MT, CV, JA, JM, AB, BG, RC, JPQ, GP, NA, AWT and JPF contributed for drafting the work, revising it critically for important intellectual content and approved the final version of the manuscript. All authors gave their agreement to be accountable for all aspects of the work, and ensure the accuracy and integrity of any part of the work.

Funding The study was funded by 'Fondation de France', 'AADAIRC' and 'Association Sports et Collection'. The study promoter is the University Hospital of Poitiers, Poitiers, France.

Competing interests AWT reports financial support (payment for lectures and travel expenses coverage to attend scientific meetings) by Fisher \& Paykel, Covidien, Maquet - Getinge, GE Healthcare. J-PF reports consulting fees from Fisher \& Paykel and SOS Oxygène.

Patient and public involvement Patients and/or the public were not involved in the design, or conduct, or reporting, or dissemination plans of this research.

Patient consent for publication Not required.

Provenance and peer review Not commissioned; externally peer-reviewed.

Data availability statement No data are available. Data will be handled according to French law. Coding participants will be done by recording the first letter of the name and forename, accompanied by a single study identifier indicating the order of participant inclusion, in order to store anonymised data in the electronic case report form. The sponsor will ensure that each study participant has given his/her consent for access to his/her personal data that is strictly required for quality control of the study. All investigators will have access to the final data set. Investigators will make available the documents and individual data strictly required for monitoring, quality control and audit of the study to persons having access to them, in accordance with the statutory and regulatory provisions in place (articles L.1121-3 and R.5121-13 of the French Public Health Code).

Open access This is an open access article distributed in accordance with the Creative Commons Attribution Non Commercial (CC BY-NC 4.0) license, which permits others to distribute, remix, adapt, build upon this work non-commercially, and license their derivative works on different terms, provided the original work is properly cited, appropriate credit is given, any changes made indicated, and the use is non-commercial. See: http://creativecommons.org/licenses/by-nc/4.0/.

ORCID iDs

René Robert http://orcid.org/0000-0001-5989-5409

Erwan l'her http://orcid.org/0000-0002-3341-7086

\section{REFERENCES}

1 Rochwerg B, Brochard L, Elliott MW, et al. Official ERS/ATS clinical practice guidelines: noninvasive ventilation for acute respiratory failure. Eur Respir J 2017;50. doi:10.1183/13993003.02426-2016. [Epub ahead of print: 3108 2017].

2 Curtis JR, Cook DJ, Sinuff T, et al. Noninvasive positive pressure ventilation in critical and palliative care settings: understanding the goals of therapy. Crit Care Med 2007;35:932-9.

3 Kacmarek RM. Should noninvasive ventilation be used with the do-not-intubate patient? Respir Care 2009;54:223-9. discussion 229-231.

4 Azoulay E, Demoule A, Jaber S, et al. Palliative noninvasive ventilation in patients with acute respiratory failure. Intensive Care Med 2011;37:1250-7.

5 Benhamou D, Girault C, Faure C, et al. Nasal mask ventilation in acute respiratory failure. experience in elderly patients. Chest 1992;102:912-7.

6 Azoulay E, Kouatchet A, Jaber S, et al. Noninvasive mechanical ventilation in patients having declined tracheal intubation. Intensive Care Med 2013;39:292-301.

7 Nava S, Ferrer M, Esquinas A, et al. Palliative use of non-invasive ventilation in end-of-life patients with solid tumours: a randomised feasibility trial. Lancet Oncol 2013;14:219-27.

8 Frat J-P, Thille AW, Mercat A, et al. High-Flow oxygen through nasal cannula in acute hypoxemic respiratory failure. $N$ Engl $J$ Med 2015;372:2185-96.

9 Frat J-P, Ragot S, Girault C, et al. Effect of non-invasive oxygenation strategies in immunocompromised patients with severe acute respiratory failure: a post-hoc analysis of a randomised trial. Lancet Respir Med 2016;4:646-52.

10 Coudroy R, Jamet A, Petua P, et al. High-Flow nasal cannula oxygen therapy versus noninvasive ventilation in immunocompromised patients with acute respiratory failure: an observational cohort study. Ann Intensive Care 2016;6:45.

11 Sklar MC, Mohammed A, Orchanian-Cheff A, et al. The impact of high-flow nasal oxygen in the immunocompromised critically ill: a systematic review and meta-analysis. Respir Care 2018;63:1555-66. 
12 Mokart D, Geay C, Chow-Chine L, et al. High-Flow oxygen therapy in cancer patients with acute respiratory failure. Intensive Care Med 2015;41:2008-10.

13 Azoulay E, Lemiale V, Mokart D, et al. Effect of high-flow nasal oxygen vs standard oxygen on 28-day mortality in immunocompromised patients with acute respiratory failure. JAMA 2018;320:2099-107.

14 Organized jointly by the American Thoracic Society, the European Respiratory Society, the European Society of Intensive Care Medicine, and the Société de Réanimation de Langue Française, and approved by ATS Board of Directors, December 2000. International consensus conferences in intensive care medicine: noninvasive positive pressure ventilation in acute respiratory failure. Am J Respir Crit Care Med 2001;163:283-91.

15 Turnbull AE, Ruhl AP, Lau BM, et al. Timing of limitations in life support in acute lung injury patients. Crit Care Med 2014;42:296-302.

16 Meduri UG, Fox RC, Abou-Shala N, et al. Noninvasive mechanical ventilation via face mask in patients with acute respiratory failure who refused endotracheal intubation. Crit Care Med 1994;22:1584-90.

17 Chu C-M, Chan VL, Wong IWY, et al. Noninvasive ventilation in patients with acute hypercapnic exacerbation of chronic obstructive pulmonary disease who refused endotracheal intubation. Crit Care Med 2004;32:372-7.

18 Levy M, Tanios MA, Nelson D, et al. Outcomes of patients with donot-intubate orders treated with noninvasive ventilation*. Crit Care Med 2004;32:2002-7.

19 Schettino G, Altobelli N, Kacmarek RM. Noninvasive positive pressure ventilation reverses acute respiratory failure in select "donot-intubate" patients. Crit Care Med 2005;33:1976-82.

20 Cuomo A, Delmastro M, Ceriana P, et al. Noninvasive mechanica ventilation as a palliative treatment of acute respiratory failure in patients with end-stage solid cancer. Palliat Med 2004;18:602-10.

21 Duchateau F-X, Beaune S, Ricard-Hibon A, et al. Prehospital noninvasive ventilation can help in management of patients with limitations of life-sustaining treatments. Eur J Emerg Med 2010;17:7-9.

22 Meert A-P, Berghmans T, Hardy M, et al. Non-Invasive ventilation for cancer patients with life-support techniques limitation. Supportive Care in Cancer 2006;14:167-71.

23 Fernandez R, Baigorri F, Artigas A. Noninvasive ventilation in patients with "do-not-intubate" orders: medium-term efficacy depends critically on patient selection. Intensive Care Med 2007;33:350-4.
24 Bülow H-H, Thorsager B. Non-Invasive ventilation in do-not-intubate patients: five-year follow-up on a two-year prospective, consecutive cohort study. Acta Anaesthesiol Scand 2009;53:1153-7.

25 Corral-Gudino L, Jorge-Sánchez RJ, García-Aparicio J, et al. Use of noninvasive ventilation on internal wards for elderly patients with limitations to respiratory care: a cohort study. Eur J Clin Invest 2011:41:59-69.

26 Peters SG, Holets SR, Gay PC. High-Flow nasal cannula therapy in do-not-intubate patients with hypoxemic respiratory distress. Respir Care 2013;58:597-600.

27 Epstein AS, Hartridge-Lambert SK, Ramaker JS, et al. Humidified high-flow nasal oxygen utilization in patients with cancer at Memorial Sloan-Kettering cancer center. J Palliat Med 2011;14:835-9.

28 Hui D, Morgado M, Chisholm G, et al. High-Flow oxygen and bilevel positive airway pressure for persistent dyspnea in patients with advanced cancer: a phase II randomized trial. J Pain Symptom Manage 2013;46:463-73.

29 Koyauchi T, Hasegawa H, Kanata K, et al. Efficacy and tolerability of high-flow nasal cannula oxygen therapy for hypoxemic respiratory failure in patients with interstitial lung disease with Do-NotIntubate orders: a retrospective single-center study. Respiration 2018;96:323-9.

30 Zemach S, Helviz Y, Shitrit M, et al. The use of high-flow nasal cannula oxygen outside the ICU. Respir Care 2019;64:1333-42.

31 Wilson ME, Majzoub AM, Dobler CC, et al. Noninvasive ventilation in patients with Do-Not-Intubate and Comfort-Measures-Only orders: a systematic review and meta-analysis. Crit Care Med 2018;46:1209-16.

32 Gaudry S, Messika J, Ricard J-D, et al. Patient-important outcomes in randomized controlled trials in critically ill patients: a systematic review. Ann Intensive Care 2017;7:28.

33 Antonelli M, Pennisi MA, Conti G. New advances in the use of noninvasive ventilation for acute hypoxaemic respiratory failure. European Respiratory Journal 2003;22:65s-71.

34 Schmidt M, Boutmy-Deslandes E, Perbet S, et al. Differential perceptions of noninvasive ventilation in intensive care among medical caregivers, patients, and their relatives: a multicenter prospective Study-The PARVENIR study. Anesthesiology 2016;124:1347-59.

35 Thille AW, Reynaud F, Marie D, et al. Impact of sleep alterations on weaning duration in mechanically ventilated patients: a prospective study. Eur Respir J 2018:51:1702465.

36 Vilaça M, Aragão I, Cardoso T, et al. The Role of Noninvasive Ventilation in Patients with "Do Not Intubate" Order in the Emergency Setting. PLoS One 2016;11:e0149649. 